AperTO - Archivio Istituzionale Open Access dell'Università di Torino

\title{
An Innovative Model to Predict Pediatric Emergency Department Return Visits
}

\section{This is a pre print version of the following article:}

Original Citation:

Availability:

This version is available http://hdl.handle.net/2318/1609134

since 2019-07-31T11:01:48Z

Published version:

DOI:10.1097/PEC.0000000000000910

Terms of use:

Open Access

Anyone can freely access the full text of works made available as "Open Access". Works made available under a Creative Commons license can be used according to the terms and conditions of said license. Use of all other works requires consent of the right holder (author or publisher) if not exempted from copyright protection by the applicable law. 
This is the author's final version of the contribution published as:

Bergese, Ilaria; Frigerio, Simona; Clari, Marco; Castagno, Emanuele; de Clemente, Antonietta; Ponticelli, Elena; Scavino, Enrica; Berchialla, Paola. An Innovative Model to Predict Pediatric Emergency Department Return Visits. PEDIATRIC EMERGENCY CARE. None pp: 1-6.

DOI: 10.1097/PEC.0000000000000910

The publisher's version is available at:

http://content.wkhealth.com/linkback/openurl?sid=WKPTLP:landingpage\&an=00006565-900000000-98867

When citing, please refer to the published version.

Link to this full text:

http://hdl.handle.net/ 
An Innovative Model to Predict Pediatric Emergency Department Return Visits

Ilaria Bergese, RN, MNS, ${ }^{*}$ Simona Frigerio, RN, MNS, PhD, $\dagger$ Marco Clari, RN, MNS, $\dagger$

Emanuele Castagno, MD, PhD,* Antonietta De Clemente, RN, MNS, + Elena Ponticelli, RN, MNS, $\uparrow$ Enrica Scavino, RN, MNS, $\S$ and Paola Berchialla, PhD\|

*Department of Pediatric Emergency, Regina Margherita Children's Hospital of Torino

$†$ Città della Salute e della Scienza di Torino University Hospital

†Azienda Sanitaria Locale TO5, Chieri

$\S$ Cottolengo Hospital

||Department of Clinical and Biological Sciences, University of Torino, Turin, Italy.

Corresponding author

Ilaria Bergese, RN, MNS, Department of Pediatric Emergency, Regina Margherita Children's Hospital, Città della Salute e della Scienza di Torino University Hospital, Piazza Polonia 94, 10126, Turin, Italy (e-mail: ilariabergese@yahoo.it) 


\begin{abstract}
Objectives: Return visit (RV) to the emergency department (ED) is considered a benchmarking clinical indicator for health care quality. The purpose of this study was to develop a predictive model for early readmission risk in pediatric EDs comparing the performances of 2 learning machine algorithms.
\end{abstract}

Methods: A retrospective study based on all children younger than 15 years spontaneously returning within 120 hours after discharge was conducted in an Italian university children's hospital between October 2012 and April 2013. Two predictive models, artificial neural network (ANN) and classification tree (CT), were used. Accuracy, specificity, and sensitivity were assessed.

Results: A total of 28,341 patient records were evaluated. Among them, 626 patients returned to the ED within 120 hours after their initial visit. Comparing ANN and CT, our analysis has shown that CTis the best model to predict RVs. The CT model showed an overall accuracy of $81 \%$, slightly lower than the one achieved by the ANN (91.3\%), but CT outperformed ANN with regard to sensitivity ( $79.8 \%$ vs $6.9 \%$, respectively). The specificity was similar for the 2 models (CT, 97\% vs ANN, 98.3\%). In addition, the time of arrival and discharge along with the priority code assigned in triage, age, and diagnosis play a pivotal role to identify patients at high risk of RVs.

Conclusions: These models provide a promising predictive tool for supporting the ED staff in preventing unnecessary RVs. 


\section{Introduction}

T he early readmission to the emergency department (ED) within few days after discharge occurs frequently both in the adult and pediatric setting, accounting for $2.5 \%$ to $3.5 \%$ of total admission, and is considered a benchmarking clinical indicator for health care quality (1-4). Unscheduled return visits (RVs) may contribute to crowding the ED, resulting in overloading the usually busy department (5-7). In the United States, in 2013 the readmissions in ED within 1 year cost more than 1 billion dollars annually (8). The majority of studies examining the frequency and characteristics of RVs focus on the general population9 and do not provide specific information about pediatric patients, suggesting that RVs may be due to potential deficiencies in diagnosis or medical management on the first visit $(1,4,7)$. In particular, such studies on pediatric and adult patients report that the majority of RVs could be attributable to the natural progression of the disease as well as to difficulties in accessing primary care, poor patient education at discharge, and ineffective doctor/patient relationship $(1-3,10-12)$.

Many attempts have been done to prevent unnecessary adult RVs, in particular through the identification of specific patient characteristics associated with higher probability of readmission, such as time of arrival and discharge, age, insurance status, ethnicity, and clinical features.9,10,13,14 In a few studies on the pediatric population, some readmission predictors such as complex chronic clinical condition, ethnicity, and public insurance coverage have been associated with a higher risk of RV.15-17

Return visits could be preventable by also using techniques to address the quantitative risk; in particular, Lee et al7 have imple-mented software to identify specific criteria to predict RVs within 72 hours after discharge for a pediatric population, achieving an overall predictive accuracy of $80 \%$. In addition, Feudtner et al18 developed a model to predict the readmission 
of children to the hospital, with a predicted probability of readmission from $43 \%$ to $86 \%$. A wide range of these methods has been developed. The en-gineering approach is based on the idea that risk objectively exists and risk analysis is a tool to express it by probabilities and expected values.19 Among statistical and engineering approaches, there are logistic regression, artificial neural networks (ANNs), classification trees (CTs), and regression trees.19 Artificial neural networks and CT were chosen to implement an appropriate algorithm that can be trained to recognize complex data patterns. Arti-ficial neural networks and CT have been considered models of choice by many medical data classification tasks.19,20

Artificial neural networks are machine learning methods that have been described as electronic analogs of the biological ner-vous system where a large number of nervous cells are connected to each other in a complex network. Each nerve cell is linked to tens of thousands of other neurons, and the intelligent behavior emerges from the numerous interactions between the units' inter-connection. A feed-forward architecture with backpropagation learning method was implemented.21

A CT is a nonparametric method used for the hierarchical segmentation when the dependent variable is nominal. This system is able to treat qualitative and quantitative variables providing a flexible and semiautomatic model to estimate a set of classificatory rules.19

The aim of our study was to develop a predictive model for early readmission risk in pediatric EDs comparing the per-formances of 2 learning machine algorithms.

\section{Materials and Methods}

The study population consisted of children younger than 15-years-old, accessing consequently the ED of Regina Margherita University Children's Hospital, Torino, Italy, between October 2012 and April 2013, and spontaneously returning within 120 hours of discharge, according to. 
Data was collected from the hospital's computerized record system

(Trackcare1996- InterSystems, Milan, Italy) and two databases were built: one with all accesses in ED in the study period and the other one with the RVs only.

Each RV has been matched with the arrival day/time of first access and corresponding discharge day/time. Patients accessing ED within 120 hours after their initial visit were identified. All children who returned for a scheduled visit not established by the ED physician were excluded from the database.

For each RV the following variables have recorded: nationality, sex, age, day of the week (working day, Sunday and holidays), time of arrival (7,00 am-2.59 pm, 3,00 pm- 10.59 pm, 11,00 pm-6.59 am), triage color code, most common pathologies/ symptoms (fever, gastroenteritis, otitis, urinary tract infections, respiratory tract infection , bronchiolitis, pneumonia , trauma, injuries, foreign body ingestion/aspiration, neurological trouble, skin eruption, abdominal pain, general pain, epistaxis), medication prescribed and tests ordered (administered drugs, X-ray and blood test, clinical advices), mode of discharge (admission in a ward, admission in ED observation unit, discharge ) and lapse between the first and the second visit.

\section{Predictive Models}

In order to create an efficient predicting model, an ANN and a CT were implemented. Among many predictive models, ANNs and CTs were chosen since they be implemented in algorithms that can be trained to recognize complex patterns in data and they are often the models of choice in many medical data classification tasks (Berchialla et al.) and (Tseng WT, Chiang WF, Liu SY, Roan J, Lin CN. The Application of Data Mining Techniques to Oral Cancer Prognosis. Journal of Medical Systems 2015 May;39(5):59). 
The accuracy of ANN and CT models was calculated through a10-fold cross validation process by which the dataset was divided in two subgroups: a training set and a test set. The training set was then used for the learning of the model while the test set was used to assess the overall performance of ANN and CT by means of accuracy, sensitivity and specificity. To ensure the strength of the results, the cross validation process was repeated 20 times; the overall accuracy and operative characteristics were thus obtained by averaging.

\section{Artificial Neural Network}

ANNs are a machine learning method thas have been described as electronic analogues of the biological nervous system, where a large number of nervous cells are connected to each other in a complex network. Each nerve cell is linked to tens of thousands of other neurons and the intelligent behavior emerges from the numerous interactions between the units interconnected (20).

In this study, all the variables collected were used as input information (see table 1). A feedforward architecture with back-propagation learning method was implemented (Ripley, B. D. (1996). Pattern recognition and neural networks. Cambridge, Cambridge University Press.)

\section{Classification Tree}

CT is a non-parametric method used for the hierarchical segmentation when the dependent variable is nominal. This system is able jointly treat qualitative and quantitative variables providing a flexible and semiautomatic model to estimate a set of classificatory rules. In this analysis, the CT was implemented by the standard binary recursive partitioning using all predictor variables described in table 1.

All statistical analysis were performed using R version 3.02 (R Development Core Team (2012). R: A language and environment for statistical computing. Vienna, Austria.) 


\section{Results}

During the study period, 34,086 patients were evaluated. Among them, 626 patients returned to the ED within the first 120 hours after their initial visit, corresponding to a rate of $1,8 \%$. Demographic and clinical characteristics of the sample are summarized in Table 2.

The majority of patients undergoing RV were children under 2-years-old (44.1\%), males (55\%) and Italian (78.6\%). These occurred from Monday to Friday (69\%), mainly between 7.00 am and $3.00 \mathrm{pm}(48 \%)$ within 13 to 24 hours since the first visit (21\%). The most frequently observed disorders were fever, respiratory illness, infections and neurological disorders.

Figure 1 shows Artificial Neural Network with the different variables and their weight influencing the output RV. Each variable is associated to its weight or intensity. The ANN model achieved $91.3 \%$ of accuracy with a $98.3 \%$ of specificity and $6.9 \%$ of sensitivity.

The CT model is showed in Figure 2. The CT model shows $81 \%$ of accuracy, $79.8 \%$ of sensitivity in recognizing early $\mathrm{RV}$ and $97 \%$ of specificity.

From figure 2, for classifying RVs, the CT model divides patients into in two groups based on pathology/symptoms-codes: group A, which is made of codes $2,3,4,5,6)$ and group B (codes 1,8,9,10). Group A was further split into two subgroups according to triage color code separating patients with white, green or red codes from those with yellow code. Then, discharge time became discriminant. Indeed, some patients who returned in the morning $(7.00 \mathrm{am}-3.00 \mathrm{pm})$ were further split from those who returned in the afternoon and evening $(3.00 \mathrm{pm}-7.00 \mathrm{am})$. Finally the arrival time become discriminant. Patients with yellow code were removed in accordance to the age. 
Patients with trauma/ injuries were only 9 out of 626 , while those with RV for endocrinological disorder, general follow up or disease different from the other categories (skin, gynecological, urological, heart disorder) still showed high heterogeneity and were divided again based on arrival time, discharge time and triage color code.

\section{Discussion}

In this study, we have identified patients with probable RVs to our pediatric ED within 12 to 120 hours after the first visit in the same setting through the application of 2 statistical models, ANN and CT. In our analysis, RV rate within 72 hours was $1.4 \%$, lower than in another Italian study (2.5\%) reported by Costabel et al22 and also lower than the study by Alessandrini et al in Philadelphia (3.5\%) and by Goldman et al23 (5.2\%) in Toronto, but similar to the one reported by Gallagher et al13 (1.2\%) at the Boston Children's Hospital. Our data show that children aged 2 years return frequently $(44.1 \%)$ in line with the results of Alessandrini et al. Previous reports found more RVs in children younger than 1 year $(1,22)$ probably because preschool children have diminished communication skills and may not be able to express their discomfort and also because of their par-ents' poor reserve to handle illness. In fact, the parents of in-fants might be more apprehensive maybe because they have no experience with their offspring's diseases, and physicians recommend them to return to the ED more often than the par-ents of older children if they had symptoms. In contrast with the other Italian study (22), foreign children did not have a higher risk of RVs: more than $70 \%$ of our RVs were Italian, so probably our RVs were not due to communication difficulties. This result contrasts with the study of Gallagher et al that described the population with limited English proficiency as the one mainly returning to the ED. Repeated admission occurred mostly on working days. Such result differs from the other Italian study by Costabel et al,22 who observed most of RVs in the weekend. In our setting, this may be due to a deficiency in 
the primary care health system. Some parents may not trust their primary care physician or are unable to take off from work to bring their children to the primary care office for a reevaluation (2).

In-deed, the time of the day reflects difficulties with primary care because they came back mostly on daytime between 7:00 A.M. and 3:00 P.M. A great majority of RVs took place in March. This finding is not similar to previous studies reporting the winter season as the most represented, $(4,5)$ realistically because in winter, there is a higher risk to contract infectious diseases and also be-cause ED physicians spend less time on teaching at discharge be-cause of the overcrowding of EDs. Most of our RVs were due to infectious diseases like in other studies $(1,2,4,12,22)$.

Such illnesses are common in the pediatric population and sometimes have unpredictable courses (12) with respiratory complications. Previous studies showed that children with RVs had a higher likelihood to stay almost overnight on their second access; in our study, $26 \%$ of children were admitted in a hospital ward. We cannot make a conclusion regarding the causes of an increased admission rate in $\mathrm{RVs}$, but the most probable is the increased severity of the illness even if in our population, triage acuity increased at RVs only in $11 \%$ of cases. Other causes may be the lack of postdischarge supervision on account of poor compliance of the parents and, not the least, the difficulty in communicating with the general practitioner $(4,22,24)$.

Forty-five children were put on antibiotic therapy on their first visit. These children came back to the ED within 72 hours complaining that there was no therapeutic effect. This suggests that there is, likely, a lack of education by the health professionals about the drugs' effects and latency. Such RVs are probably avoidable and represent an inappropriate use of health care resources as demonstrated by Gallagher et al.13 
In our research, we introduced 2 predictive models based on machine learning algorithms to assess the risk of RV to ED within 120 hours from discharge. Feudtner et al19 have studied RVs in a pediatric ED using logistic regression. Similarly, Reinke et al15 have used logistic regression to analyze patients' discharge; the same approach was adopted by Bardach et al8 measuring the quality of care and pediatric RVs. In our research, the methodological choice of using ANNs and CTs was motivated because usually, even if logistic regression achieved greater accuracy, it is outperformed by $\mathrm{CT}$ and $\mathrm{ANN}$ when comparing other perfor-mance measures like sensitivity and specificity.19 Seven demo-graphic and clinical variables were entered into the models: time to arrival and discharge time, ethnicity, diagnosis code, age, and sex were entered according to several authors.1,4,5,10,13-17,21,23,25 In accordance with Lee et al,7 we considered the triage color code. Lee et al7 also considered the transport used to arrive at the hospi-tal as relevant information related to the hospital environment as well as the number of patients in the ED and the number of avail-able beds at the patient arrival, which were not entered in our models because they were not available. Finally, among potential variables, we did not consider the payer status7,17,23,26 or the type of insurance2,3,11,15,16,24,25 because the Italian Health Care System provides universal coverage and free ED access. Comparing ANN and CT, our analysis has shown that CT is the best model to predict RVs. The CT model showed an overall accuracy of $81 \%$, slightly lower than the one achieved by the ANN, but it outperformed ANN in sensitivity $(79.8 \%$ vs $6.9 \%$ ). Besides the performance measure, CT is popular for the easy interpretability of the output, contrary to ANNs, which provide models with difficult interpretation.19 The CT's flowchart shows that children with RVs are mostly those with diagnosis code (respiratory disease, fever, infection, pain, neurological problem) who were admitted with yellow triage color code and younger than 2 years. Such children are followed by those with the same diagnosis, but have a white, green, and red color code who arrived in the afternoon or at night 
and were discharged in the morning. In this study, the time of arrival and discharge along with the priority code played a pivotal role according to both ANN and CT. Indeed, the CT model also recognized the age as an important characteristic along with the type of diagnosis. Our study suggests that a child younger than 2 years being discharged with RV indicators (ie, with yellow triage code, fever, respiratory or infectious problems, pain or neurologic disorders) had a greater probability to return to the ED within 120 hours. This may suggest to ED cli-nicians to play out a strategy to reduce RVs, for example, by involving the nursing staff in managing discharge of such patients with particular attention to the communication in the effort of patient education.

The limits of our study are that the findings came from the retrospective analysis of the data of a single, although large, pediatric hospital. In addition, the time of observation was relatively short and the inclusion of late fall and winter might have generated a hypothetical bias linked to seasonal diseases.

\section{CONCLUSION}

In conclusion, our study shows that in the effort to reduce RVs, there are several steps that can be attempted by the ED staff. Although perfect efficiency and accuracy are not always possible in the prevention of emergency care, ANNs and CT models pro-vide a promising predictive tool focusing on the importance of using management and communicative strategy for those patients with higher risk of RVs. 


\section{BIBLIOGRAFIA}

1) Cho C S, Shapiro D J, Cabana M D, Maselli J H, Hersh A L. A national depiction of children with return visits to the emergency department within 72 hours, 2001-2007. Pediatr Emerg care 2012; 28: 606-10

2) Adekoya N. Patients seen in emergency departments who had a prior visit within the previous 72 h- National hospital ambulatory medical care survey, 2002. Public Health 2005; 119:914-18.

3) Costabel S, Piccotti E, Sartini M, Magnani M, Di Piero P. Return visits to the paediatric emergency department: first analysis in Italy. J Prev Med Hyg 2008; 49: 142-47.

4) Berry J G, Toomey S L, Zaslavsky A M, Jha A K, Nakamura M M, Klein D J et all. Pediatric readmission prevalence and variability across hospital. Jama 2013; 309: 372-80

5) Easter J S, Bachur R. Physicians' assessment of pediatric returns to the emergency department. Emerg Med J 2013; 44: 682-88.

6) LeDuc K, Rosebrook H, Rannie M, Gao D. Pediatric emergency department recidivism: demographic characteristics and diagnostic predictors. Int Emerg Nurs 2006; 32: 131-38.

7) Alessandrini E A, Lavelle J M, Grenfell S M, Jacobstein C R, Shaw K N. Return visits to a pediatric emergency department. Pediatr Emerg Care 2004; 20: 166-71.

8) Ali A B, Place R, Howell J Malubay S M. Early pediatric emergency department return visits: a prospective patient- centric assessment. Clin Pediatr 2012; 51: 651-58.

9) Abualenain J, Frohna W J, Smith M, Pipkin M, Webb C, Milzman D. The prevalence of quality issues and adverse outcomes among 72-hours return admissions in the emergency department. J Emerg Med 2013; 45: 281-88 
10) Lawrence L M, Jenkins $C$ A, Zhou C, Given T G. The effect of diagnosis-specific computerized discharge instructions on 72-hour return visits to the pediatric emergency department. Pediatr Emerg Care 2009; 25:733-38.

11) Gaucher N, Bailey B, Gravel J. For children leaving the emergency department before being seen by a physician, counseling from nurses decreases return visits. Int Emerg Nurs 2011; 19: $173-77$

12) Lee E K, Yuan F, Hirsh D, Mallory M D, Simon H K. A clinical decision tool for predicting patient care characteristics: patients returning within 72 hours in the emergency department. Amia Annu Symp Proc 2012; 2012: 495-04.

13) Gallagher R A, Porter S, Monuteaux M C, Stack A M. Unscheduled return visits to the emergency department the impact of language. Pediatr Emerg Care 2013; 29: 579-83

14) Goldman R D, Kapoor A, Mehta S. Children admitted to the hospital after returning to the emergency department within 72 hours. Pediatr Emerg Care 2011; 27: 808-11.

15) Timm N L, Mona L H, Luria J W. Pediatric emergency department overcrowding and impact on patient flow outcomes. Acad Emerg Med 2008; 15: 832-37

16) Goldman R D, Ong M, Macpherson A. Unscheduled return visits to the pediatric emergency department-one year experience. Pediatr Emerg Care 2006; 22: 545-49.

17) Bardach N S, Vittinghoff E, Asteria- Penalza R, Edwards J D, Yazdany J, Lee H C et all. Measuring hospital quality using pediatric readmission and revisit rates. Pediatrics 2013; 132: $429-36$

18) Berry J G, Hall D E, Kuo D Z, Cohen E, Agrawal R, Feudtner C et all. Hospital utilization and characteristics of patients experiencing recurrent readmissions within children's hospital. Jama 2011; 305: 682-90 
19) Feudtner C, Levin J E, Srivastava R, Goodman D M, Slonin A D, Sharma V et all. How well can hospital readmission be predicted in a cohort of hospitalized children? A retrospective multi-centre study. Pediatrics 2009; 123: 286-93

20) H. Bandy. Developing a neural network system, AAII Working Paper,1997

21) Berchialla P, Scarinzi C, Snidero S, Gregori D. Comparing models for quantitative risk assessment: an application to the european registry of foreign body injuries in children. Stat Methods Med Res 2013;0:1-16

22) Breiman L, Friedman J H, Olshen R A, Stone C J. Classification and regression trees. New York: Chapman and Hall, 1984

23) Sun Y, Heng B H, Tay S Y, Seow E. predicting hospital admission at emergency department triage using routine administrative data. Acad Emerg Med 2011;18:844-49

24) Reinke D A, Walker M, Boslaugh S, Hodge D. Predictors of pediatric emergency patients discharged against medical advice. Clin Pediatr 2011;48:263-70 
Table 1. Category values used for each variables entered in the predictive models.

\begin{tabular}{|l|l|}
\hline Variable & Category values \\
\hline Arrival time & $\begin{array}{l}1(7.00 \mathrm{am}-2.59 \mathrm{pm}) ; 2(3.00 \mathrm{am}-10.59 \mathrm{pm} 3(11.00 \mathrm{pm}-06.59 \\
\mathrm{am})\end{array}$ \\
\hline Discharge time & $\begin{array}{l}1(07.00 \mathrm{am}-2,59 \mathrm{pm}) ; 2(3.00 \mathrm{pm}-10.59 \mathrm{pm}) ; 3(11,00 \mathrm{pm}- \\
06.59 \mathrm{am})\end{array}$ \\
\hline Sex & 1 (male); (female) \\
\hline Age & $0-15$ years \\
\hline Triage color code & 1 (white); (green); 3 (yellow); 4 (red) \\
\hline Nationality & 1 (Italian); (foreign patient) \\
\hline Pathologies/ Symptoms & $1-10 *$ \\
\hline
\end{tabular}

*1 trauma and injuries, 2 respiratory disease, 3 fever, 4 infection, 5 pain, 6/7 neurological and oncological problem, 8 endocrinological disorder, 9 follow up (established by the ED), 10 miscellaneous category ( skin problem, urological, cardiological disorder) 
Table 2. Sample characteristics at first visit and return visit. P-values were computed using chi-square test or Fisher's exact test when the expected value of a cell was less than five.

\begin{tabular}{|c|c|c|c|}
\hline & I visit & Return Visit & $\mathrm{p}$-value \\
\hline \multicolumn{4}{|l|}{ Gender } \\
\hline Male & $19,139(56.1 \%)$ & $344(55 \%)$ & 0.57 \\
\hline Female & $14,497(43.9 \%)$ & $282(45 \%)$ & \\
\hline \multicolumn{4}{|l|}{ Age } \\
\hline $0-2$ & $13,210(38.7 \%)$ & $276(44.1 \%)$ & $<0.001$ \\
\hline 3-6 years & $9,290(27.2 \%)$ & $180(28.8 \%)$ & \\
\hline $7-10$ years & $6,416(18.8 \%)$ & $78(12.5 \%)$ & \\
\hline $11-15$ years & $5,170(15.1 \%)$ & $92(14.7 \%)$ & \\
\hline \multicolumn{4}{|l|}{ Nationality } \\
\hline Italian & $27,953(82 \%)$ & $492(78.6 \%)$ & 0.03 \\
\hline Non Italian & $6,133(18 \%)$ & $134(21.4 \%)$ & \\
\hline \multicolumn{4}{|l|}{ Triage code } \\
\hline White & $5.078(14.7 \%)$ & $12(1.9 \%)$ & $<0.001$ \\
\hline Green & $19,827(58.5 \%)$ & $435(69.5 \%)$ & \\
\hline Yellow & $9,126(26.7 \%)$ & $176(28.1 \%)$ & \\
\hline Red & $55(0.2 \%)$ & $3(0.5 \%)$ & \\
\hline \multicolumn{4}{|l|}{ Pathologies/ symptoms } \\
\hline Trauma /injuries & $9.816(28.7 \%)$ & $9(1.4 \%)$ & $<0.001$ \\
\hline respiratory disease & $7,293(21.5 \%)$ & $188(30 \%)$ & \\
\hline infection & $6,542(19.1 \%)$ & $205(32.7 \%)$ & \\
\hline pain & $3,596(10.7 \%)$ & $78(12.5 \%)$ & \\
\hline $\begin{array}{l}\text { fever } \\
\text { neurological// }\end{array}$ & $2,288(6.7 \%)$ & $14(2.2 \%)$ & \\
\hline endocrinological & $1,275(3.7 \%)$ & $43(6.9 \%)$ & \\
\hline follow up & $640(1.9 \%)$ & $8(1.3 \%)$ & \\
\hline endocrinological disorder & $269(0.8 \%)$ & $2(0.3 \%)$ & \\
\hline miscellaneus category & $2,367(6.9 \%)$ & $79(12.6 \%)$ & \\
\hline
\end{tabular}




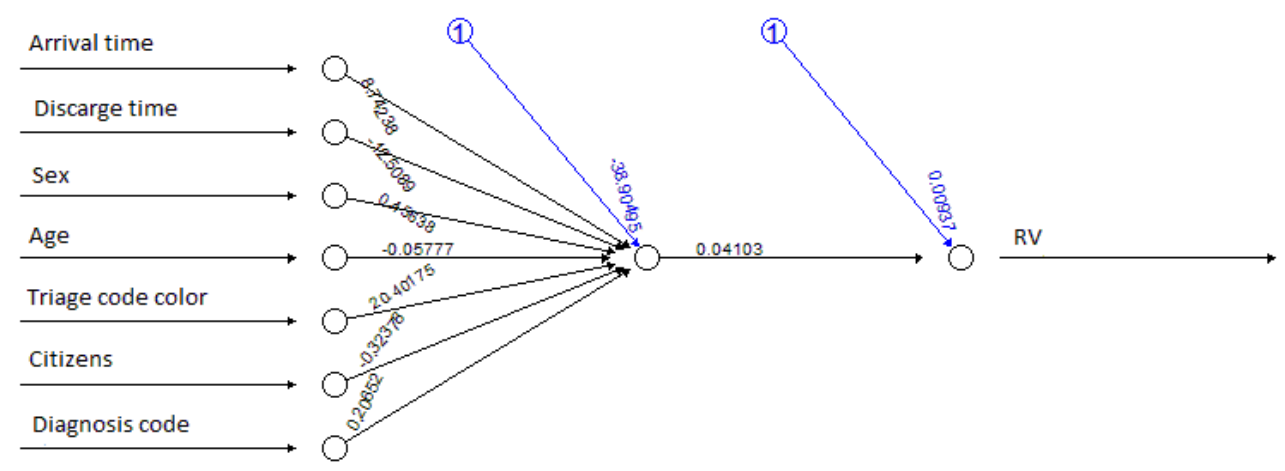

Figure 1. ANN's architecture. The white nodes on the left represent the input variables, which are transformed into a numerical output (RV node on the right) that represent the RV value (0: no RV; $1: \mathrm{RV}$ ), after they have been weighted through the activation of a non-linear function represented by the intermediate node. 



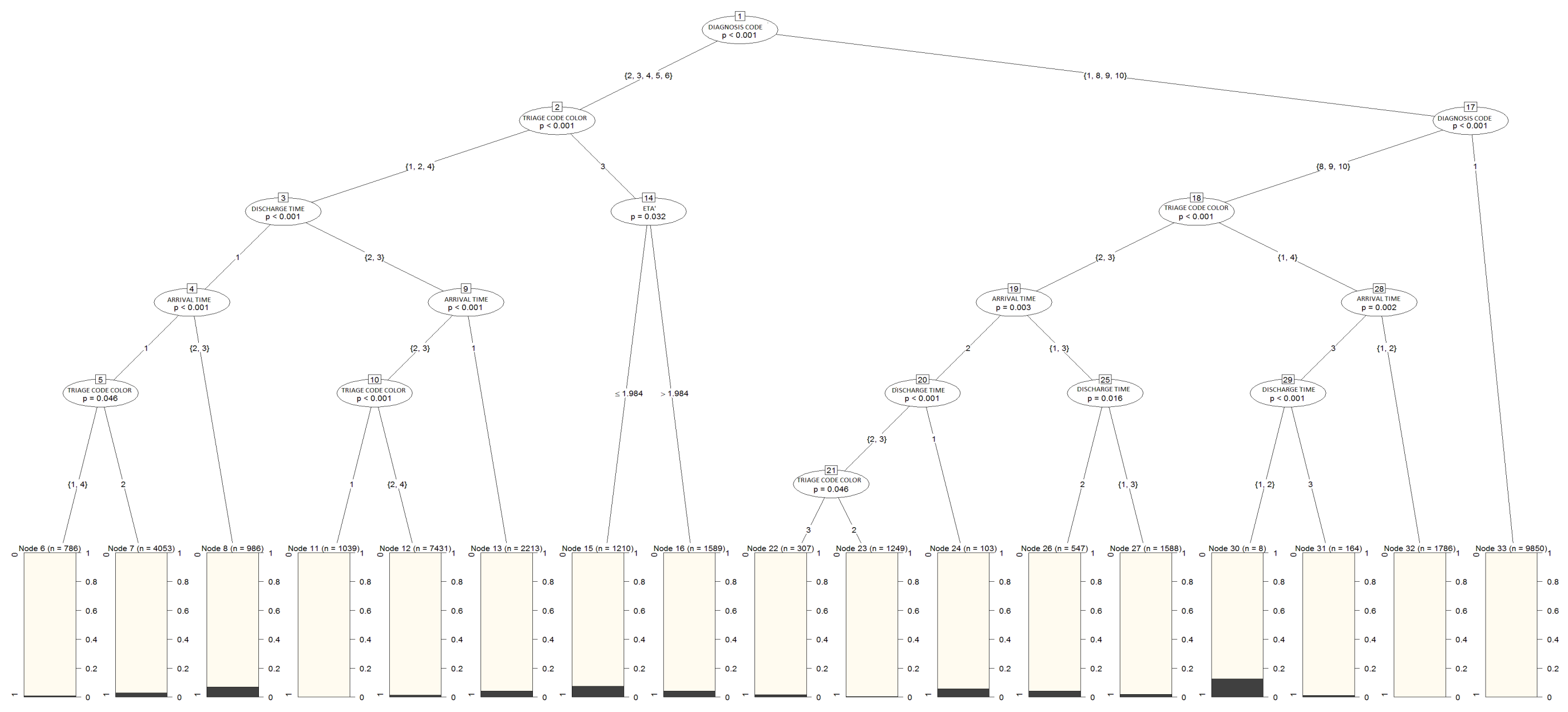

Figure 2. The rule based process identified by the classification tree to classify RVs. Predictor variables are show inside white ovals, barplots at the bottom give the percentage of RVs for each group. 
